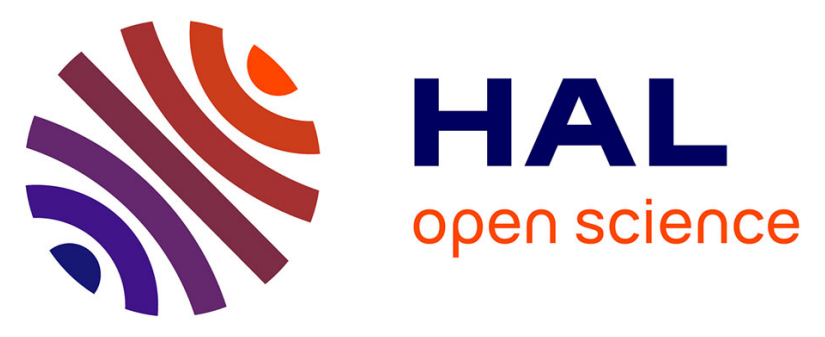

\title{
Abundant molecular oxygen in the coma of comet 67P/Churyumov-Gerasimenko
}

Andre Bieler, Kathrin Altwegg, Hans Balsiger, Akiva Bar-Nun, Jean-Jacques Berthelier, P. Bochsler, Christelle Briois, Ursina Calmonte, M. Combi, J. de Keyser, et al.

\section{To cite this version:}

Andre Bieler, Kathrin Altwegg, Hans Balsiger, Akiva Bar-Nun, Jean-Jacques Berthelier, et al.. Abundant molecular oxygen in the coma of comet 67P/Churyumov-Gerasimenko. Nature, 2015, 526 (7575), pp.678-681. 10.1038/nature15707 . hal-01346075

\section{HAL Id: hal-01346075 \\ https://hal.science/hal-01346075}

Submitted on 20 Jul 2016

HAL is a multi-disciplinary open access archive for the deposit and dissemination of scientific research documents, whether they are published or not. The documents may come from teaching and research institutions in France or abroad, or from public or private research centers.
L'archive ouverte pluridisciplinaire HAL, est destinée au dépôt et à la diffusion de documents scientifiques de niveau recherche, publiés ou non, émanant des établissements d'enseignement et de recherche français ou étrangers, des laboratoires publics ou privés. 
1 Measurement of molecular oxygen in the coma of 67P/Churyumov-Gerasimenko 2

3 Authors: A. Bieler ${ }^{1,}{ }^{2 *}$, K.Altwegg $^{2,}{ }^{3}$, H. Balsiger ${ }^{2}$, A. Bar-Nun ${ }^{4}$, J.-J. Berthelier ${ }^{5}$, P. Bochsler ${ }^{2}$, C.

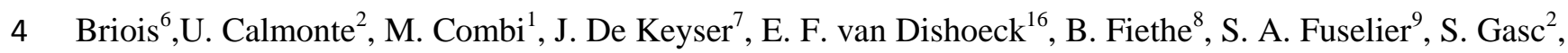
5 T. I. Gombosi ${ }^{1}$, K. C. Hansen ${ }^{1}$, M. Hässig ${ }^{2,9}$, A. Jäckel ${ }^{2}$,E. Kopp ${ }^{2}$, A. Korth ${ }^{10}$, L. Le Roy ${ }^{3}$, U. Mall ${ }^{10}$, B. 6 Marty $^{11}$, O. Mousis ${ }^{12}$, T. Owen ${ }^{13}$, H. Rème ${ }^{14,}{ }^{15}$, M. Rubin ${ }^{1}$, T. Sémon ${ }^{2}$, C.-Y. Tzou ${ }^{2}$, J. H. Waite ${ }^{9}$, C. 7 Walsh $^{16}$, P. Wurz ${ }^{2,3}$

\section{Affiliations:}

$9{ }^{1}$ Department of Atmospheric, Oceanic and Space Sciences, University of Michigan, 2455 Hayward Street, 10 Ann Arbor, MI 48109, USA

$11 \quad{ }^{2}$ Physikalisches Institut, University of Bern, Sidlerstr. 5, CH-3012 Bern, Switzerland.

$12{ }^{3}$ Center for Space and Habitability, University of Bern, Sidlerstr. 5, CH-3012 Bern, Switzerland.

$13{ }^{4}$ Department of Geoscience, Tel-Aviv University, Ramat-Aviv, Tel-Aviv, Israel

$14{ }^{5}$ LATMOS/IPSL-CNRS-UPMC-UVSQ, 4 Avenue de Neptune F-94100, Saint-Maur, France.

$15{ }^{6}$ Laboratoire de Physique et Chimie de l'Environnement et de l'Espace (LPC2E), UMR 6115 CNRS 16 Université d'Orléans, France.

$17{ }^{7}$ Belgian Institute for Space Aeronomy, BIRA-IASB, Ringlaan 3, B-1180 Brussels, Belgium.

$18{ }^{8}$ Institute of Computer and Network Engineering (IDA), TU Braunschweig, Hans-Sommer-Straße 66, D1938106 Braunschweig, Germany.

$20{ }^{9}$ Department of Space Science, Southwest Research Institute, 6220 Culebra Rd., San Antonio, TX 78228, 21 USA.

$22{ }^{10}$ Max-Planck-Institut für Sonnensystemforschung, Justus-von-Liebig-Weg 3, 37077 Göttingen, Germany.

$23{ }^{11}$ Centre de Recherches Pétrographiques et Géochimiques, CRPG-CNRS, Université de Lorraine, 15 rue 24 Notre Dame des Pauvres, BP 20, 54501 Vandoeuvre lès Nancy, France.

$25{ }^{12}$ Aix Marseille Université, CNRS, LAM (Laboratoire d'Astrophysique de Marseille) UMR 7326, 13388, 26 Marseille, France. 
${ }^{13}$ Institute for Astronomy, University of Hawaii, Honolulu, HI 96822, USA

${ }^{14}$ Université de Toulouse; UPS-OMP; IRAP, Toulouse, France.

${ }^{15}$ CNRS; IRAP; 9 Avenue du Colonel Roche, BP 44346, F-31028 Toulouse Cedex 4, France.

${ }^{16}$ Leiden Observatory, Leiden University, P.O. Box 9513, 2300 RA Leiden, Netherlands.

\section{Abstract}

The composition of the neutral gas coma of a comet is dominated by $\mathrm{H}_{2} \mathrm{O}, \mathrm{CO}$ and $\mathrm{CO}_{2}$, typically comprising as much as $95 \%$ of the total gas density ${ }^{1}$. In addition to these common species, the cometary coma has been measured to contain a rich array of additional molecules including noble gases, sulfuric compounds and complex hydrocarbons. Molecular oxygen $\left(\mathrm{O}_{2}\right)$, despite its detection on other icy bodies, such as the moons of Jupiter and Saturn ${ }^{2,3}$, had however remained undetected in the cometary volatile inventory. Here we report the direct in situ measurement of molecular oxygen in the cometary coma of 67P/Churyumov-Gerasimenko with a local abundance ranging from $1 \%$ to $10 \%$ relative to $\mathrm{H}_{2} \mathrm{O}$ and a mean value of $3.7 \pm 1.5 \%$. Our observations indicate that the $\mathrm{O}_{2} / \mathrm{H}_{2} \mathrm{O}$ ratio is isotropic in the coma anddoes not systematically change over a period of several months. This suggeststhat $\mathrm{O}_{2}$ was incorporated into the cometary nucleus primordially during the comet's formation. Current Solar System formation models do not predict conditions that would allow this to occur, suggesting that our detection may play a significant role in advancing our understanding of comet formation and the prevailing conditions and processes in the early stages of our Solar System.

Measurements of the coma of 67P/Churyumov-Gerasimenko (hereafter 67P) were made between September 2014 and March 2015 with the ROSINA-DFMS mass spectrometer ${ }^{4}$ onboard the Rosetta spacecraft. For this study we analyzed 2808 mass spectra taken in this time period. Due to the high resolving power and sensitivity of ROSINA-DFMS it is possible to unambiguously differentiate between the three main species present in the narrow mass range centered on $32 \mathrm{Da} / \mathrm{e}$; molecular oxygen $\left(\mathrm{O}_{2}\right)$, sulfur $(\mathrm{S})$ and methanol $\left(\mathrm{CH}_{3} \mathrm{OH}\right)$, something which has not been achieved byprevious in situ or remote measurements at comets. Fig. 1shows several measurements centered at the $\mathrm{O}_{2}$ peak. The green and orange lines show data taken before the close encounter with 67P. Only minorsignaturesfrom the tenuous neutral gas atmosphere of theRosetta spacecraft can be identified and even after long thruster firing maneuvers, which use $\mathrm{N}_{2} \mathrm{O}_{4}$ as an oxidizer, the contamination of the $\mathrm{O}_{2}$ signal remains small (green line in Fig. 1).Measurements while orbiting 67P, shown as the light blue, dark blue and purple line in Fig. 1 , show a clear increase of the $\mathrm{O}_{2}$ signal, indicating the presence of cometary $\mathrm{O}_{2}$. These three measurements were taken at decreasing distances $(r)$ from the comet nucleus and follow the predicted $1 / r^{2}$ signal dependencethat is expected for a conserved cometaryspecies, further gaining confidence in our detection.

As previously reported, the local number densities in the coma vary spatially and temporally, ${ }^{5,6}$, for different compounds. The bottom panel of Fig. 2 shows an $\mathrm{O}_{2}$ and $\mathrm{H}_{2} \mathrm{O}$ measurement sequence taken between the $5^{\text {th }}$ and the $7^{\text {th }}$ of November 2014 and the peaks and valleys occur with the rotation frequency of 12.4 hours of $67 \mathrm{P}^{7}$. During this time range the radial distance from the comet is nearly constant at roughly $30 \mathrm{~km}$, the phase angle decreases from 115 to 102 degrees, the latitude linearly (in time) increases from -22 to 22 degrees and all longitudes are covered multiple times (due to the short 
rotation of 67P). Uncertainties of the individual measurements for both species are on the order of $30 \%$ and are indicated by the shaded areas in Fig. 2. We observe a strong correlation between the absolute abundances of $\mathrm{H}_{2} \mathrm{O}$ and $\mathrm{O}_{2}$, not only for the time span shown in Fig. 2, but for the entire dataset.This strong correlation, with a Pearson correlation coefficient of 0.91 , indicates that $\mathrm{H}_{2} \mathrm{O}$ and $\mathrm{O}_{2}$ are of similar origin on the nucleus and their release mechanismsarelinked. Despite the overall strong correlation the $\mathrm{O}_{2}$ ratio decreases for high $\mathrm{H}_{2} \mathrm{O}$ abundances as can be seen in the top panel of Fig. 2 . Because of the radial dependence of the number density there is however not an absolute valueabove which this saturation effect is observed. This effect is the main cause for the variability in the measured $\mathrm{O}_{2}$ ratios as shown in the time series in Fig 3.

A plausible mechanism for the strong $\mathrm{O}_{2} / \mathrm{H}_{2}$ Ocorrelation is the possibility toproduce $\mathrm{O}_{2}$ by radiolysis or photolysis of water ice. Here we follow the nomenclature that photolysis refers to UV photons that break bonds, whereas radiolysis refers to more energetic photons or fast electrons and ions depositing energy into the ice and ionizing molecules. Creation of sputtered $\mathrm{O}_{2}$ by radiolysis was demonstrated by Hart et al. $^{8}$ and is observed for the icy moons of Jupiter; Europa, Ganymede and Callisto ${ }^{9-11-}$, as well as for the moons of Saturn; Dione and Rhea ${ }^{3}$.At comets, radiolysis does happen on different time scales:during billions of years while they are residing in the Kuiper belt, over the period of several years once they enter the inner Solar System and on very short time scales.In the first case, the skin depth for producing $\mathrm{O}_{2}$ is in the range of a meter, while in the latter it is only a few micrometers. Once a comet begins its residence in the inner Solar System, it loses several meters of its surface material during each orbit around the Sun, therefore we can safely assume that all radiolysis products created in the Kuiper Belt phase are gone from 67P. Furthermore, radiolysis and photolysis by solar wind and UV radiationin the inner Solar Systemonly affect the top few micrometers of the cometary surface. Due to 67P's continuous mass loss through outgassing, we estimate the surface loss to be in the range of several $\mathrm{cm}$ for the time from August 2014 to March 2015. If recent production were the source or the measured $\mathrm{O}_{2}$, our data would show a continuous decrease of the $\mathrm{O}_{2}$ ratio over the examined time period.Apart from the variations related to the $\mathrm{H}_{2} \mathrm{O}$ abundance, Fig. 3 shows that we do not observe a systematic change in the $\mathrm{O}_{2}$ ratio over several months. On line creation of the measured $\mathrm{O}_{2}$ by radiolysis or photolysis at 67P seems overall unlikely and would lead to anisotropic $\mathrm{O}_{2}$ ratios. Given that radiolysis and photolysis, on any of the discussed time scales, do not seem plausible production mechanisms for $\mathrm{O}_{2}$, our preferred explanationis the incorporation of primordial $\mathrm{O}_{2}$ into the cometary nucleus.

Time dependent models of surface grain chemistry in molecular clouds predict an abundanceof a few percent of $\mathrm{O}_{2}$ relative to $\mathrm{H}_{2} \mathrm{O}$ on icy grains at timescalesof $>10^{6}$ years ${ }^{12}$. Thereby $\mathrm{O}_{2}$ is formed together with $\mathrm{H}_{2} \mathrm{O}$ by grain surface reactions, whichwould explain their correlation and be in line with the very low abundances of $\mathrm{HO}_{2}$ and $\mathrm{H}_{2} \mathrm{O}_{2}$ that we measured in the coma of 67P (see Fig. 4). A further consequence would bethat these icy grains have been incorporated into the comet mostly unaltered, a fact very much under debate, but which has recently been proposed again by Cleeves et al. ${ }^{13}$ and would also be in accordance with the measured $\mathrm{D} / \mathrm{H}$ ratio in $67 \mathrm{P}^{14}$.

Constraints on the $\mathrm{O}_{2}$ abundance are of great benefit for future theoretical studies and modeling efforts as the current understanding of the grain-gas interaction is still evolving.In contrast, laboratory experiments of photolysis or particle bombardment of $\mathrm{H}_{2} \mathrm{O}$ ice find significantly higherabundances of $\mathrm{H}_{2} \mathrm{O}_{2}$ than we measured ${ }^{15}$.

Interestingly, there has not been adirect detection of $\mathrm{O}_{2}$ in interstellar ices, but upper limits for the $\mathrm{O}_{2} / \mathrm{H}_{2} \mathrm{O}$ ratiosof $<0.1$ have been published based on analysis of solid ${ }^{13} \mathrm{CO}$ lines ${ }^{16,17}$ on icy grains. These 
limits then lead to a $\mathrm{O}_{2} / \mathrm{CO}_{2}$ ratio of approximately 0.75 . Both ofthese ratios, albeit relatively uncertain, agree with our findings and previous observations of $67 \mathrm{P}^{2}$.So far, this non-detection has preferably been explained by the high volatility and chemical reactivity of $\mathrm{O}_{2}$. Thispotential $\mathrm{O}_{2}$ abundance on icy grainson the order of a few percent relative to $\mathrm{H}_{2} \mathrm{O}$ would have significant implications on our current understanding of ices in interstellar molecular clouds and their importance for the formation of comets. The global distribution of elemental $\mathrm{O}$ in the interstellar medium is probably not affected by our findings, as $\mathrm{O}_{2}$ on ice grains with an abundance of a few percent relative to $\mathrm{H}_{2} \mathrm{O}$ accountsonly for a small fraction of the total $O$ inventory.

An alternative explanation for the $\mathrm{O}_{2}$ is the incorporation of gaseous $\mathrm{O}_{2}$ into water ice. Modelsof protoplanetary disks do show that $\mathrm{O}_{2}$ can be abundant in the comet forming zone. A rapid cooling scenario from $>100 \mathrm{~K}$ to less than $30 \mathrm{~K}$ is then needed to form water ice with trapped $\mathrm{O}_{2}$. However, despite great efforts by remote sensing campaigns, $\mathrm{O}_{2}$ has only been detected intwo regions in the interstellar medium so far ${ }^{18-20}$. This lack of $\mathrm{O}_{2}$ is not understood but as a consequence, molecular oxygenis generally considered to be present only at very low abundances. However, the abundance ratios of $\mathrm{HO}_{2} / \mathrm{O}_{2}$ and $\mathrm{H}_{2} \mathrm{O}_{2} / \mathrm{O}_{2}$ determined by DFMS for the coma of $67 \mathrm{P}$ are very close to those observed in the rho Oph $\mathrm{A}$ interstellar core, one of the two regions where $\mathrm{O}_{2}$ was actually detected in the ISM. We find ratios of $\mathrm{HO}_{2} / \mathrm{O}_{2}=(1.9 \pm 0.3) \times 10^{-3}$ and $\mathrm{H}_{2} \mathrm{O}_{2} / \mathrm{O}_{2}=(0.6 \pm 0.07) \times 10^{-3}$, which are similar to $\mathrm{HO}_{2} / \mathrm{O}_{2}$ $\mathrm{H}_{2} \mathrm{O}_{2} / \mathrm{O}_{2} \sim 0.6 \times 10^{-3}$ as reported for rho Oph $\mathrm{A}^{21,22}$. To what extent these gas-phase abundance ratios reflect those in the ice is however still unclear.

The unique case of rho Oph A has been interpreted by having experienced slightly higher temperatures of around $20-30 \mathrm{~K}$ over its lifetime, compared to $\sim 10 \mathrm{~K}$ for most other interstellar clouds ${ }^{22,23}$. Applied to our own Solar System this might indicate it was formed from an unusual warm molecular cloud, challenging our current understanding of the chemistry occurring during these early stages. Admittedly, the observed ratios are in contrast to simulations of interstellar ice chemistry, which predict $\mathrm{H}_{2} \mathrm{O}_{2}$ and $\mathrm{O}_{3}$ ice to be more abundant than $\mathrm{O}_{2}$ ice by one to two orders of magnitude ${ }^{24}$. We found no evidence for the presence of ozone $\left(\mathrm{O}_{3}\right)$ (see Fig. 4) for which we give an upper limit of $1 \times 10^{-6}$ relative to water.

\section{References}

[1] Bockelée-Morvan, D., Mumma, M. J., Weaver, H. A. 2004, In Comets II, University of Arizona Press, Tucson, Arizona, p. 391-423.

[2] Lanzerotti, L. J., Brown, W. L., Poate, J. M. \& Augustyniak, W. M. Production of $\mathrm{O}_{2}$ on icy satellites by electronic excitation of low-temperature water ice. Geophys. Res. Lett. 5, 155-158 (1978).

[3] Noll, K. S., Roush, T. L., Cruikshank, D. P., Johnson, R. E. \& Pendleton, Y. L. Detection of ozone on Saturn's satellites Rhea and Dione. Nature 388, 45-47 (1997).

[4] Balsiger, H. et al. ROSINA - ROSETTA orbiter spectrometer for ion and neutral analysis. Sp. Sci. Rev. $128,745-801,(2007)$ 
158

159

160

161

162

163

164

165

166

167

168

169

170

171

172

173

174

175

176

177

178

179

180

181

182

183

184

185

186

187

188

189

190

191

192

193

194

195

196

197

198

199

200

201
[5]Hässig, M. et al. Time variability and heterogeneity in the coma of 67P/Churyumov-Gerasimenko. Science 347 (2015). URL http://www.sciencemag.org/content/347/6220/aaa0276.abstract.http://www.sciencemag.org/content/ 347/6220/aaa0276.full.pdf.

[6] Adrienne

[7] ref on rotation period

[8] Hart, E. G. \& Platzmann, R. L. Mechanisms in Radiobiology (Academic Press, New York, 1961).

[9] Carlson, R. W. et al. Hydrogen peroxide on the surface of Europa. Science 283, 2062-2064 (1999).

URL http://www.sciencemag.org/content/283/5410/2062.abstract.

http://www.sciencemag.org/content/283/5410/2062.full.pdf.

[10] Spencer, J. R., Calvin, W. M. \& Person, M. J. Charge-coupled device spectra of the Galilean satellites: Molecular oxygen on Ganymede. J. Geophys. Res. (1995).

[11] Spencer, J. R. \& Calvin, W. M. Condensed $\mathrm{O}_{2}$ on Europa and Callisto. The Astronomical Journal 124, 3400-3403 (2002).

[12] D’Hendecourt, L. B, Allamandola, L. J., Greenberg, J. M. Time dependent chemistry in dense molecular clouds. I - Grain surface reactions, gas/grain interactions and infrared spectroscopy. Astron. Astrophys., 152, 130-150, (1985)

[13] Cleeves, L. I., Bergin, E. A., Alexander, C. M., Du, F., Graninger, D., Öberg, K. I., Harries, T. J. The ancient heritage of water ice in the solar system. Science, 345, (2014)

[14] Altwegg et al. 67P/Churyumov-Gerasimenko, a Jupiter family comet with a high D/H ratio. Science, 347, (2015)

[15] Zheng, W., Jewitt, D. \& Kaiser, R. I. Formation of hydrogen, oxygen and hydrogen peroxide in electron-irradiated crystalline water ice. The Astrophysical Journal 639, 534-548 (2006).

[16] Vandenbussche, B. et al. Constraints on the abundance of solid $\mathrm{O}_{2}$ in dense clouds from ISO-SWS and ground-based observations. Astron. Astrophys., 346, L57-L60 (1999)

[17] Pontoppidan, K. et al. A 3-5 $\mu \mathrm{m} \mathrm{m}$ VLT spectroscopic survey of embedded young low mass stars I. Structure of the CO ice. Astron. Astrophys., 408, 981-1007 (2003)

[18] Liseau, R. et al. Multi-line detection of $\mathrm{O}_{2}$ toward rho Ophiuchi A. Astron. Astrophys., 541, A73 (2012) 
202

203

204

205

206

207

208

209

210

211

212

213

214

215

216

217

218

219

220

221

222

223

224

225

226

227

228

229

230

231

232

233

234

235

236

237

238

239

240

241

[19] Larsson, B., Liesau, R., Pagani, L. et al. Molecular oxygen in the Ophiuchi cloud. Astron. \& Astrophys., 466, 999 (2007)

[20] Goldsmith, P. F., Liesau, R., Bell, T. A. et al. Herschel Measurements of molecular oxygen in Orion. ApJ, 737, 96, (2011)

[21] Bergman, P. et al. Detection of interstellar hydrogen peroxide, Astron. Astrophys., 531, L8 (2011)

[22] Parise, B., Bergman, P., Du, F. Detection of the hydroperoxyl radical $\mathrm{HO}_{2}$ toward rho Ophiuchi A. Additional constraints on the water chemical network. Astron. Astrophys., 541, L11 (2012)

[23] Du, F., Parise, B., Bergman, P. Production of interstellar hydrogen peroxide $\left(\mathrm{H}_{2} \mathrm{O}_{2}\right)$ on the surface of dust grains. Astron. Astrophys., 538, A91 (2012)

[24] Taquet, V., Ceccarelli, C., Kahane, C.Multilayer modeling of porous grain surface chemistry. I. The GRAINOBLE model. Astron. Astrophys., 538, A42 (2012) 


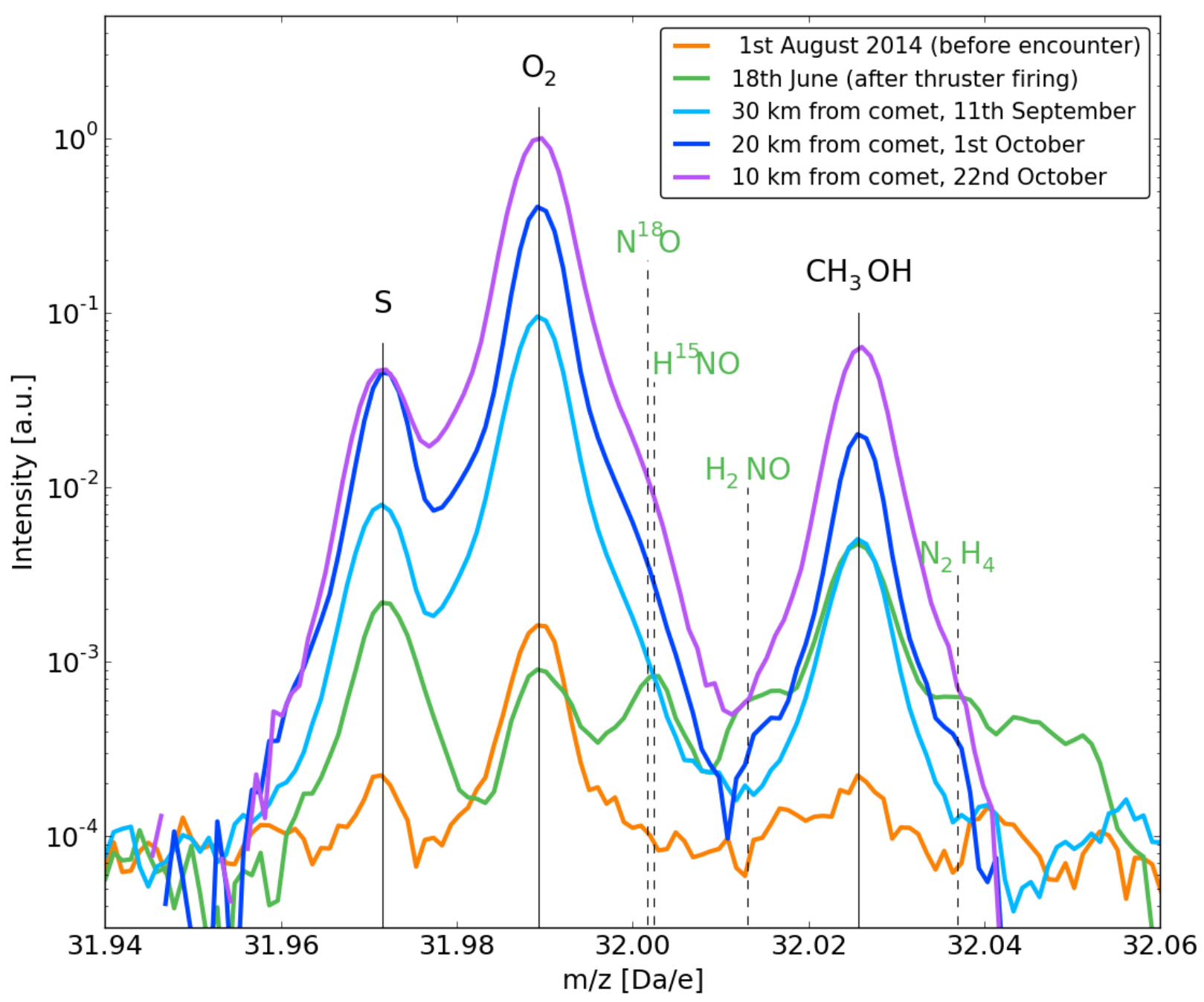

Figure 1: Normalized DFMS mass spectrum at $32 \mathrm{Da} / \mathrm{e}$. The black labels indicate the three major species found in the coma of 67P at $32 \mathrm{Da} / \mathrm{e}$. The green labels and graph identify contamination peaks from thruster firings, the contribution to the $\mathrm{O}_{2}$ peak is very low. The light blue, dark blue and purple lines represent measurements taken at different distances from the comet nucleus. 

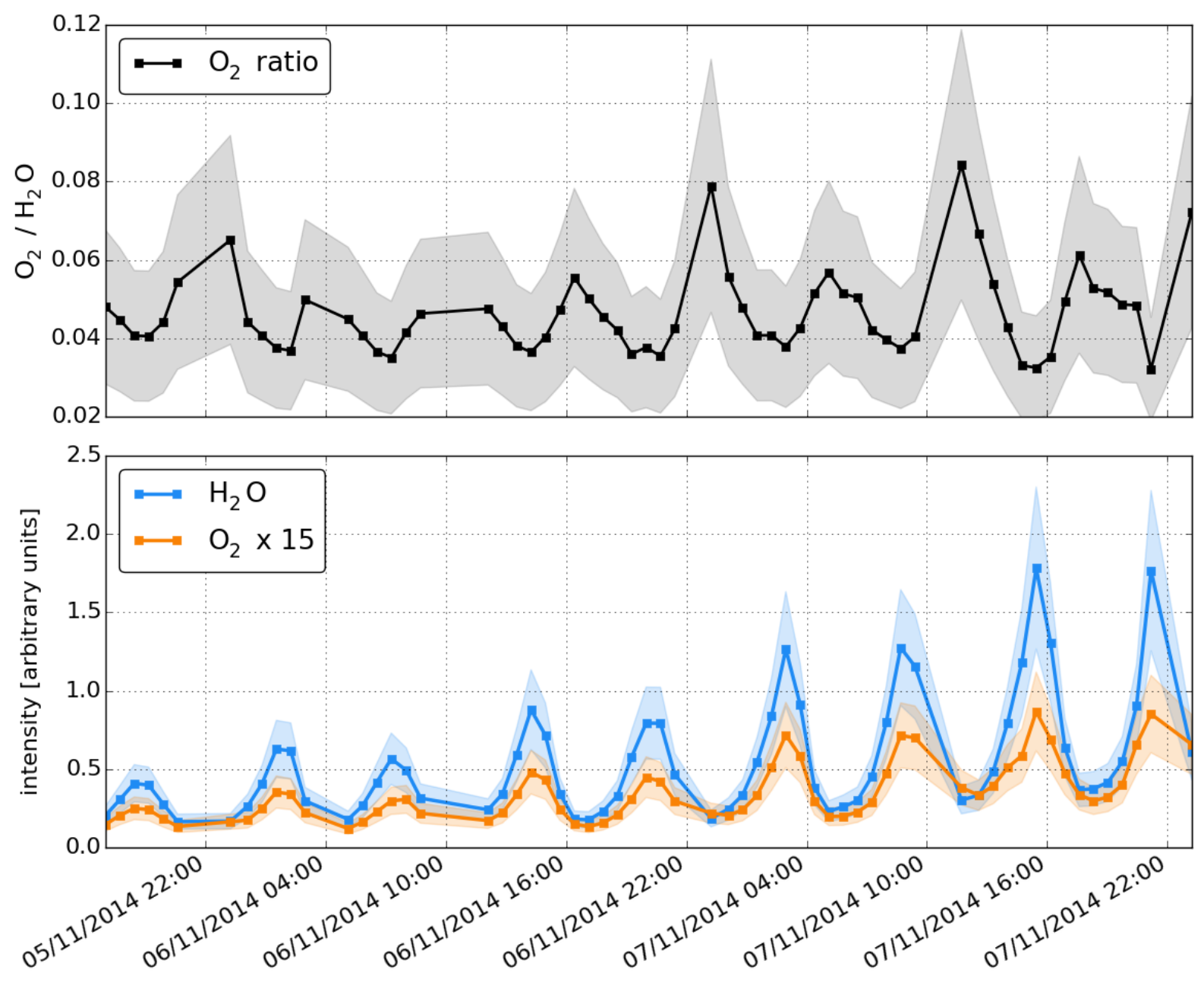

Figure 2: Correlation between $\mathrm{O}_{2}$ ratio and the $\mathrm{H}_{2} \mathrm{O}$ abundance for $5^{\text {th }}$ to $7^{\text {th }}$ of November 2014. Shaded areas indicate 1 sigma error limits. The $\mathrm{O}_{2}$ signal in the bottom panel is multiplied by a factor of 15 for visual clarity. The $\mathrm{O}_{2}$ ratio systematically drops for high $\mathrm{H}_{2} \mathrm{O}$ abundances. 


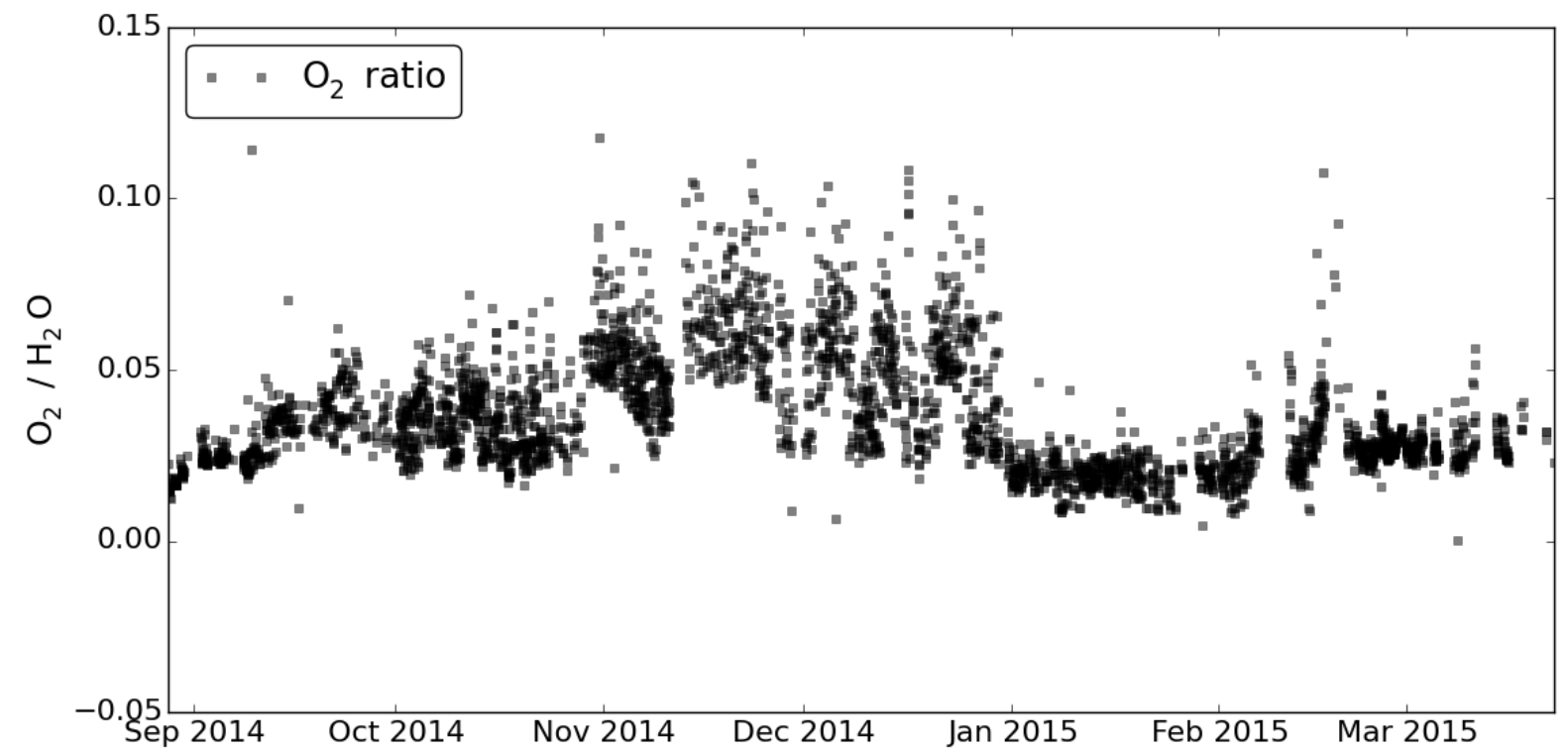

269

270

271

272

273

274

275

276

277

278

279

280

281

282

283

284

285

286

287

Figure 3: $\mathrm{O}_{2}$ ratio over time. There seems to be no systematic increase or decrease of the $\mathrm{O} 2$ ratio, as the variances can be explained by Figure 2.
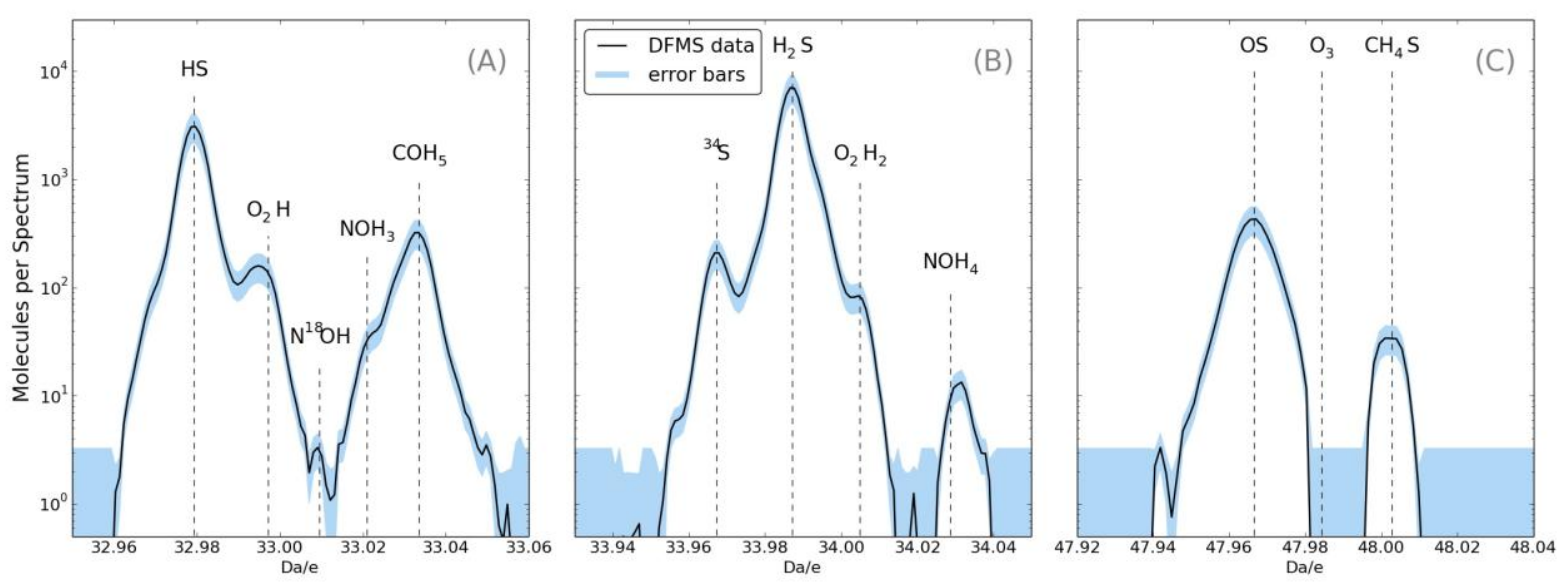

Figure 4: DFMS spectra for some of the common products of radiolysis of water ice; $\mathrm{O}_{2}, \mathrm{O}_{2} \mathrm{H}, \mathrm{O}_{2} \mathrm{H}_{2}$ and $\mathrm{O}_{3}$. This data was recorded on October $20^{\text {th }}$ at around 01:00 UTC. With the exception of $\mathrm{O}_{3}$ (see panel C), all the previously mentioned species are measured and can clearly be identified in the mass spectra of DFMS. Shaded areas mark 1 sigma error boundaries. 


\section{Methods}

\section{Spacecraft outgassing background}

To clearly identify the measured $\mathrm{O}_{2}$ as cometary in origin, all non-cometary sources of $\mathrm{O}_{2}$ must be considered and excluded. The Rosetta spacecraft produces a neutral gas cloud of its own, mainly due to diffusion of volatiles out of spacecraft material and desorption of re-deposited volatiles from the spacecraft. For example, by changing the spacecraft attitude, different components are illuminated by the Sun, which then warm up and release gas. The orange line in Fig. 1 shows the low level signals from this spacecraft contamination for $\mathrm{O}_{2}, \mathrm{~S}$, and $\mathrm{CH}_{3} \mathrm{OH}$, referred to as "background", in measurements taken several days before the encounter with 67P. It is not possible to distinguish this background signal from any potential cometary signature with DFMS, but it has been well characterized prior to the arrival at 67P and is usually orders of magnitude lower than the measured $\mathrm{O}_{2}$ signals ${ }^{6}$. To keep the background influence as low as possible, we only considered mass spectra where both the $\mathrm{O}_{2}$ and $\mathrm{H}_{2} \mathrm{O}$ abundances are at least 10 times larger than the corresponding spacecraft contamination. Another potential source of $\mathrm{O}_{2}$ is the oxidizer, $\mathrm{N}_{2} \mathrm{O}_{4}$, used by the Rosetta spacecraft during thruster firings. Measurements taken shortly after a large thruster firing maneuver from June 2014 (still before arrival at the comet) show minor contaminations around $32 \mathrm{Da} / \mathrm{e}$, but not directly affecting the $\mathrm{O}_{2}$ peak (see green curve in Fig. 1). Although contamination from thruster firings is small, DFMS measurements are usually performed hours after thruster firings, in order to minimize influence thereof. Finally we can exclude the production of $\mathrm{O}_{2}$ inside the instrument through a careful review of all oxygen-bearing molecules up to $150 \mathrm{Da} / \mathrm{e}$, which could potentially fragment into $\mathrm{O}_{2}$ in the DFMS electron impact ion source. Many minor species contain $\mathrm{O}_{2}$ but these are too low in abundance to account for the large amount of $\mathrm{O}_{2}$ detected. The remaining possibility is $\mathrm{CO}_{2}$, which is very abundant in the coma of $67 \mathrm{P}^{2}$. However, due to its chemical structure it only fragments into $\mathrm{CO}$ and $\mathrm{O}$, not $\mathrm{O}_{2}{ }^{[7]}$. Finally, we exclude the production of $\mathrm{O}_{2}$ from $\mathrm{H}_{2} \mathrm{O}$ in the instrument. For 81 mass spectra taken from May to the end of June 2014 we determine an $\mathrm{O}_{2}$ abundance of $(0.18 \pm 0.07) \%$ relative to $\mathrm{H}_{2} \mathrm{O}$, which is a factor of 20 lower than the cometary values.

\section{Correlation with $\mathrm{H}_{2} \mathrm{O}$}

The measured $\mathrm{O}_{2}$ signal shows a very strong dependence on radial distance $(\mathrm{r})$ from the comet. It increases by roughly one order of magnitude when the radial distance from the cometdecreases from 30 $\mathrm{km}$ to $10 \mathrm{~km}$. This is in agreement with a predicted $1 / \mathrm{r}^{2}$ dependence of the number density profile of a non-reactive species. Examining the data further, we observe a strong correlation between $\mathrm{H}_{2} \mathrm{O}$ and 
$\mathrm{O}_{2}$ (see Fig. 2, Pearson correlation coefficient $\mathrm{R}=0.91$ ) for data from September 2014 to March 2015. This correlation indicates that $\mathrm{O}_{2}$ and $\mathrm{H}_{2} \mathrm{O}$ are both of a similar cometary origin. In contrast, there is no correlation between $\mathrm{O}_{2}$ and $\mathrm{H}_{2} \mathrm{O}$ for measurements taken before the arrival at the comet $(\mathrm{R}=-0.01)$. The observed temporal variations in the $\mathrm{O}_{2} / \mathrm{H}_{2} \mathrm{O}$ ratio are largely due to a non-linear correlation between $\mathrm{H}_{2} \mathrm{O}$ and $\mathrm{O}_{2}$ for high water densities. The $\mathrm{O}_{2}$ ratio drops with increasing $\mathrm{H}_{2} \mathrm{O}$ abundance as is indicated in Fig. 2. A possible explanation for this is a modification of the ice-dust matrix close to the surface, e.g. sintering or re-deposition of ice grains as surface frost which then is depleted in $\mathrm{O}_{2}$. The correlation similarly supports the ruling out of $\mathrm{CO}_{2}$ as a source of the $\mathrm{O}_{2}$ because Hässig et al. ${ }^{2}$ and Rubin et al. ${ }^{8}$ show a lack of correlation between the abundance of $\mathrm{H}_{2} \mathrm{O}$ and other species like $\mathrm{CO}, \mathrm{CO}_{2}$ and $\mathrm{N}_{2}$.

\section{Radiolysis}

The production of $\mathrm{O}_{2}$ from water ice by radiolysis is the result of several reactions, where initially $\mathrm{H}, \mathrm{O}$, and $\mathrm{OH}$ are produced, followed by subsequent rearrangement to form $\mathrm{H}_{2}, \mathrm{HO}_{2}$ and $\mathrm{H}_{2} \mathrm{O}_{2}$ and ultimately $\mathrm{O}_{2}{ }^{[10,11]}$. These radiolysis products can either be trapped ${ }^{12}$ inside voids in the water ice that are also created by radiolysis, or can be scattered or desorbed directly from the surface

\section{Sputtering}

$\mathrm{O}_{2}$ production through surface sputtering: Wurz et al. ${ }^{21}$ show that sputtering of refractory materials from the cometary surface due to the solar wind is occurring now at 67P. This work demonstrates a clear difference between the southern and northern latitudes in the measured abundances of the sputtered species. This apparent spatial difference is explained via the asymmetry of the neutral coma, with higher number densities in the northern hemisphere that is preferentially exposed to the Sun for the time span under study. The solar wind, which is responsible for the sputtering, is therefore attenuated more efficiently by these denser parts of the coma and thus has limited or no access to the surface. We find that the $\mathrm{O}_{2} / \mathrm{H}_{2} \mathrm{O}$ ratio is independent of latitude and relatively constant over a period of 7 months. Furthermore, the major part of the top surface of $67 \mathrm{P}$ accessible by the solar wind does not contain any water ice ${ }^{22}$. This suggests that sputtering cannot be the main source of the detected molecular oxygen. Moreover, the sputter yields by solar wind ions are orders of magnitude too low to explain the observed amount of $\mathrm{O}_{2}$.

\section{Recent radiolysis}

$\mathrm{O}_{2}$ production after the comet formed: Zheng et al. ${ }^{25}$ argue that Kuiper belt objects, such as $67 \mathrm{P}$, subject to radiolysis, reach an equilibrium abundance of $\mathrm{O}_{2}$ and $\mathrm{H}_{2} \mathrm{O}$ in the surface ice layer after some $10^{5}$ years, leading to a relative abundance of $\mathrm{O}_{2} / \mathrm{H}_{2} \mathrm{O}$ of about $0.6 \%$. Once these objects enter the inner Solar System they lose (due to their activity and depending on their perihelion distance) material from the surface in the range of several meters per orbit around the Sun. Therefore, as most products built up during the stay in the Kuiper belt reside in the outer few meters, these should be released quickly on the first solar passages. A study by Maquet ${ }^{26}$ shows that $67 \mathrm{P}^{\prime}$ 's perihelion distance was within the orbit of Jupiter for the last 250 years, possibly even for more than 5000 years. Since a close encounter with Jupiter in 1959, the perihelion distance of 67P has been about $1.3 \mathrm{AU}$ with an orbital period of 6.4 years. Accumulated over the last perihelion passages we can assume that67P has lost hundreds of meters from its surface. Although Galactic Cosmic Rays with energies well above $1 \mathrm{GeV}$ can penetrate down to depths of hundreds of meters, the amount of $\mathrm{O}_{2}$ that can be produced by GCR-induced radiolysis appears to be at least several orders of magnitude too small if one assumes that the present-day high-energy GCR flux represents the average flux over the history of the Solar System.There are also uncertainties on the cross-sections, the relative proportion of high- $Z$ cosmic rays, and the role of porosity and defects in the cometary ice ${ }^{21,27}$. Overall, it appears unlikely that most of the $\mathrm{O}_{2}$ in 67P is of evolutionary origin. 